the kidneys in a very short time and dilute the pus to such an extent that it passes through the ureter without producing colic pains.

Since then I have treated numerous cases of acute cystitis with Ozonate Lithia Water, and all patients have remarked the immediate relief from painful and frequent micturition. Although I have not made chemical researches as to the secretian of urates under the treatment, there is a decided improvement experienced in all the different troubles based upon the overcharged condition of the blood with uric acid. The continuous use of this water after recovery can only have a beneficial effect, inasmuch as it would be preventing a recurrence of the disease.

A contra-indication to the extensive use of the water is the parenchymatous inflammation of the kidneys, as the Lithia, according to recent researches, is likely to stimulate the disease. On the other hand two or three quarts of the water are useful to wash out the kidneys and relieve the tubuli contorti from the presence of casts without being harmful. In cases of dropsy produced by heart disease, the use of the water ought not to be advised, as the amount of fluid embodied increases the work to be done by the diseased organ.

34 Washington Street.

\section{REPORT OF AN EPIDEMIC OF TYPHOID FEVER AND THE USE OF THE} WOODBRIDGE TREATMENT.

Read before the Portage County Medical Society, November, 1895. BY JOHN J. ORTON, M.D.

HEALTH OFFICER, RANDOLPH, OHIO.

Gentlemen:-There is a rule in rhetoric which I think is called Pope's Rule:

"In words, as fashion, the same rule will hold,

Be not the first by whom the new are tried,

This is a good rule to follow in the use of medicine as well as words. Nearly every mail brings us one or more circulars, singing the praises of some new remedy and it would be difflcult and unwise to test them all. Being naturally conservative, and remembering the teachings of one of the now deceased professors of my Alma Mater to avoid "journal practice," it was with doubt and hesitation that I began to treat typhoid fever by the "Woodbridge treatment."

During September and October, our little village of about 250 inhabitants passed through one of the worst epidemics of typhoid $f \in v e r$ in its history. Since May, 1895, twenty cases of typhoid fever have occurred in my practice. All but one, or possibly two, originated in this place. One came here sick from Cleveland, but not until toward the close of the epidemic, and one, a traveling salesman, became ill while away and came home; there is no way of telling whether his disease originated here or away, as he is of ten at home over Sunday.

Six persons have been sick with fever soon after leaving this place and five of these six have died. Three of the twenty cases under my care have died; two of typhoid fever alone, and one old gentleman of typhoid fever complicated with serious chronic diseases. At the beginning of the epidemic I searched the text-books in vain for some treatment that might prove satisfactory. All said that typhoid fever could not be shortened by a single day. Loomis, whose work on practice was the first I bought, and for whom
I entertain great respect and admiration, says: "The duty of the physician is to guide the disease, as far as he may be able, to a favorable issue and prevent injury to organs essential to life, keeping in mind that a certain definite period must elapse before this result can be accomplished."

Having read some of the articles on typhoid fever written by Dr. J. E. Woodbridge, of Youngstown, Ohio, I was favorably impressed with the results he reported from the use of his treatment, and wrote to him for instructions and obtained a supply of tablets put up by Parke, Davis \& Co. according to his formulæ. Not being satisfied with the results I obtained, I went to Youngstown to see Dr. Woodbridge personally, but found that he was in Chicago. My journey was not in vain, however, for I was very kindly treated by Dr. M. V. Cunningham, who had charge of Dr. Woodbridge's practice during his absence and who told me all I wished to learn in regard to the use of the remedies used by Dr. Woodbridge.

Since my return I have had excellent results in the treatment of typhoid fever; all of my patients have improved under this line of treatment, some of them more rapidly than I dared to hope for. I have the pleasure to-day of showing you several charts which differ from those shown in the text-books. These charts show a step-like decline to the normal tem. perature, which, in most of them, is reached in two weeks or less. On one, the normal line is touched in ten days, although there was a slight fever in the afternoon for five days longer. One of them shows the normal line touched on the twelfth day of treatment, and sixteenth day of disease, and one shows the same result on the fourteenth day of treatment and seventeenth day of illness. All of these patients had the rose spots and other symptoms necessary to establish a positive diagnosis, and three of them were seen by other physicians who confirmed my diagnosis of typhoid fever. As yet I have not been able to obtain as brilliant results as Dr. Woodbridge reports, but have found that, if the medicine is perseveringly used, the temperature will invariably decline, de. lirium, tympanitis and other alarming symptoms will disappear and an early convalescence will follow.

The medicine must be pusbed as fast as possible until the temperature falls below 100 degrees or these results will not be obtained. No. 1 should be given every fifteen minutes for twenty-four or thirty-six hours; then No. 2 every fifteen minutes to every halfhour right along until the temperature falls below 100 degrees. By the end of the first twenty-four or fortyeight hours of treatment the medicine should have produced five or six or more free evacuations of the bowels, and, should one tablet of either No. 1 or No. 2 not be sufficient to produce this effect, two or three or more may be given at each dose. The size and frequency of the dose may now be reduced so as to produce a gradual decrease in the frequency of the movements each day, uptil, by the time the temperature has touched normal, one or two free evacuations of the bowels occur during each twenty-four hours, and at no time should a day pass without this action being accomplished. About the third or fourth day No. 3 should be commenced and should be given every three or four hours until the temperature remains normal. If the temperature again rises No. 2 should be pushed energetically. Of course the patient must be closely watched, and if any symptoms of ptyalism should occur omit the tablets for a day 
or two and give a saturated solution of potassium chlorate, a teaspoonful every three hours, returning to the tablets as soon as possible.

I have met with one patient, a lady 46 years of age, who did not tolerate the mercury well. After three days' use of the tablets she suffered with slight ptyalism and sore gums but it was not very severe. I stopped No. 2 and gave the potash solution for fortyeight hours. I then gave small doses of podophyllin, gr. 1-8 to 1-6, every four hours alternating with No. 3 . She did well on this treatment. I believe that by the careful use of these remedies, or by a similar line of antiseptic treatment, more lives can be saved than by any other plan. Stimulants and tonics should be given if indicated, but with this treatment they will not be needed as often as by the older methods. Plenty of cold (not iced) boiled or distilled water should be given and the patient encouraged to take several swallows with each dose of medicine. Milk diet should be enforced and no solid food given until the temperature remains normal. If the patient has an aversion to milk, some good liquid food can be given, but milk should be taken also in smaller quantities than when used alone. The following report of two cases show how the medicine can be pushed:

E. J., age 6 years, began to complain October 20. On the evening of October 22 I saw him and found a

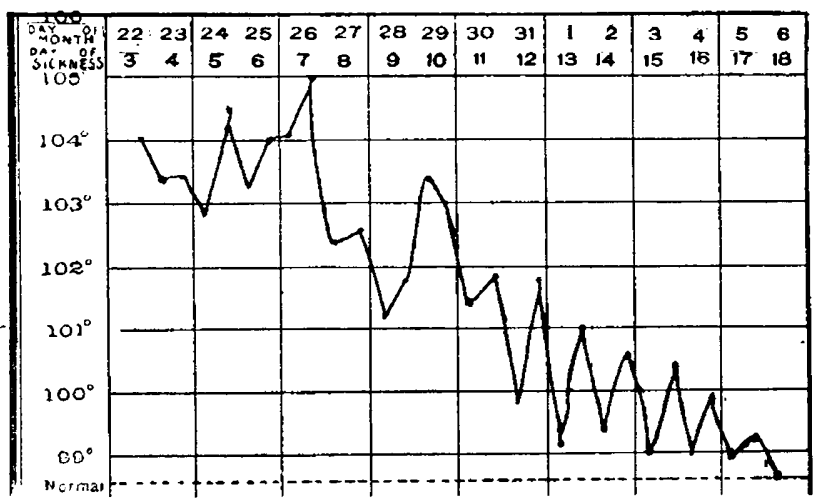

E. J., age 6 years.

temperature of 104 degrees. I gave No. 1 every half hour for thirty-six hours; then No. 2 every twenty minutes a part of the time and every half hour a part of the time until October 31 (nine days), when the temperature reached 99.8 degrees. The tablets were then given less often, and teasponnful doses of liquid peptonoids, with creasote and guaiacol, were given every three hours. This patient was so young that I did not try to give No. 3, as I feared that he could uot swallow the large capsules. On October 26 bis temperature was 105 degrees, but fell day by day till it touched normal a few hours over fourteen days of treatment, and on the seventeenth day of illness. Although this boy took the tablets very often for nearly two weeks, castor oil was frequently given to obtain sufficient movements of the bowels.

On October 1\%. Mrs. O. H., age 71 years, first felt that she was not as well as usual. She had been assisting in the care of her daughter who came from Cleveland, five weeks before, sick with typhoid fever. On October 16 she was obliged to go to bed and her temperature rose day by day till it reached 101.6 degrees on November 19. Then I gave tablets of Dr. Woodbridge's No. 1 at 9 A. M., and gave them every twenty minutes until 10 P. M.; then every half hour till 2 P. M. on the 20 th. The temperature then being only 100.5 degrees, tablets were given every half hour till 5 P. M. The temperature then having risen to nearly 101 degrees, No. 2 was given every half hour till $9 \mathrm{~A}$. M. on the 21 st. when the temperature was 99 degrees; No. 2 was given every hour till 2 P. M., then every half hour as the temperature was rising and continued till $10 \mathrm{P}$. M.; then every twenty minutes till $1 \mathrm{~A}$. M. on the $22 \mathrm{~d}$; then every half hour till 9 A. M. At 9:30 the temperature fell to 96.6 degrees, but the pulse was 82 and good. Stimulants were given and hot applications made to the feet and limbs; her temperature rose to 100.2 degrees in afternoon. The next morning the temperature fell to 97 degrees and went to 100.6 degrees in the afternoon.

The temperature was sub-normal for three days and longer, and rose slightly above normal in the afternoon gradually having less departure from normal either above or below. Tonics and stimulants were given when temperature was below and Woodbridge tablets or capsules when above. This lady took the Woodbridge medicine seven days, when the temperature remained normal, or slightly below in the

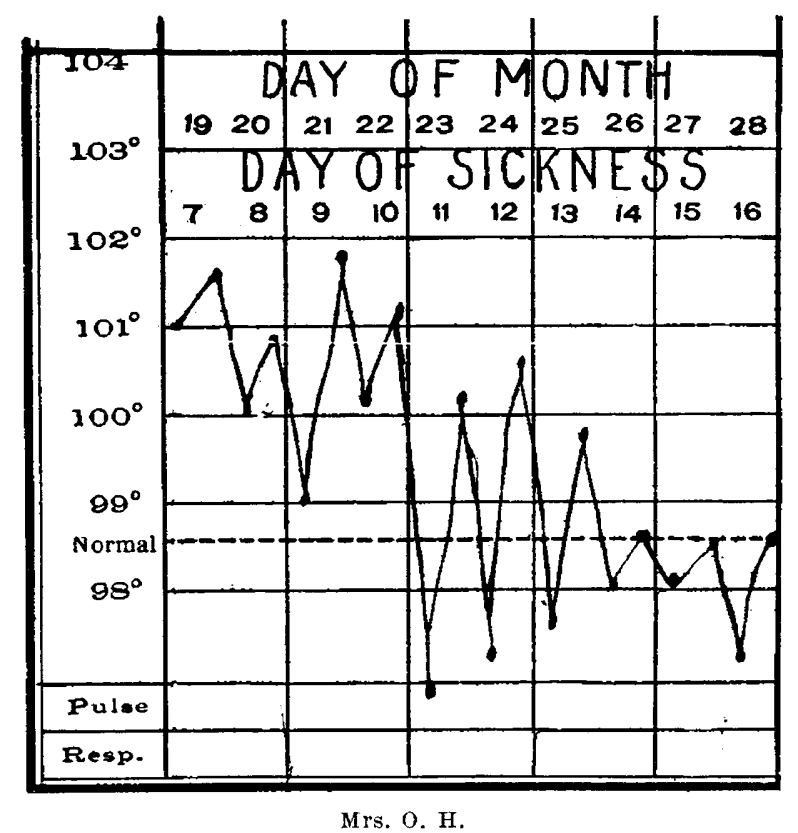

morning. She told me that she nearly always had a sub-normal temperature when recovering from any sickness. This was an interesting case to me, as most text-books say that it is extremely rare for a person in advanced years to have typhoid fever. She had rose spots, tenderness over the bowels and enough other symptoms to leave no doubt of the correctness of the diagnosis.

Eight persons received the Woodbridge treatment with no death. In every case the effect of the medicine was plainly shown by a decline in the temperature and improvement in all symptoms. Those who received the treatment early had no dry tongue and but little tympanitis or diarrhea.

Twelve patients received other treatment with three deaths. No doubt deaths will occur under any plan of treatment, but I believe that the mortality from typhoid fever would be greatly lessened if physicians would use the Woodbridge treatment. I had excellent opportunities during this epidemic to test their efficacy, aud, until something better is found, shall continue to use them in typhoid fever. 